\title{
Article
}

Mycosphere

\section{Hypogeous gasteroid Lactarius sulphosmus sp. nov. and agaricoid Russula vinosobrunneola sp. nov. (Russulaceae) from China}

\author{
Li GJ ${ }^{1,2}$, Zhang $\mathrm{CL}^{1}$, Lin $\mathrm{FC}^{1 *}$ and Zhao $\mathrm{RL}^{1,3^{*}}$
}

${ }^{1}$ State Key Laboratory for Rice Biology, Institute of Biotechnology, Zhejiang University, Hangzhou 310058, China

${ }^{2}$ State Key Laboratory of Mycology, Institute of Microbiology, Chinese Academy of Sciences, No. 1 West Beichen Rd, Chaoyang District, Beijing 100101, China

${ }^{3}$ College of Life Sciences, University of Chinese Academy of Sciences, Huairou District, Beijing 100408, China

Li GJ, Zhang CL, Lin FC, Zhao RL 2018 - Hypogeous gasteroid Lactarius sulphosmus sp. nov. and agaricoid Russula vinosobrunneola sp. nov. (Russulaceae) from China. Mycosphere 9(4), 838858, Doi 10.5943/mycosphere/9/4/9

\begin{abstract}
Two new species of Russulaceae from China are herein described and illustrated based on their morphologies and phylogenies. A hypogeous gasteroid species, Lactarius sulphosmus sp. nov. and an agaricoid species, Russula vinosobrunneola sp. nov. are introduced. The latter is morphologically distinguished from $R$. sichuanensis, although the ITS-based phylogeny was unable to distinguish them. Therefore, a multi-gene phylogenetic analysis of the $n L S U, I T S, m t S S U$, and tef-1 $\alpha$ gene sequences of Russula subsection Laricinae was carried out, which supports the assertion that they are different species.
\end{abstract}

Key words - Basidiomycota - phylogeny - Agaricomycetes - Russulales - taxonomy

\section{Introduction}

Sequestrate and angiocarpic basidiomata have frequently been observed in many groups of Agaricomycetes (Calonge \& Martín 2000, Watling \& Martín 2003, Danks et al. 2010, Henkel et al. 2010, Orihara et al. 2012, Lebel \& Vellinga 2013, Trierveiler-Pereira et al. 2014, Hongsanan et al. 2015, Li et al. 2018a). Various potential selective pressures have been proposed to explain the hymenium transformation from free or attached gills to enclosed labyrinthoid chambers and the disappearance of the stipe (Miller et al. 2001). It may be regarded as a protective mechanism in the macrofungi to prevent the effects of extreme environmental conditions, such as low temperature and aridity (Thiers 1984a, Bruns et al. 1989, Bougher \& Lebel 2001, Wilson et al. 2011). Gasteroid to secotioid basidiomata and hypogeous habitats can provide protection against moisture loss and ice gelivation (Binder \& Bresinsky 2002, Trappe \& Claridge 2005). In tropical areas, gasteromycetization of fructifications make angiocarpous species less dependent on seasonal changes. The abundance of angiocarpic basidiomata is often overlooked (Verbeken et al. 2014).

Members of Russulaceae are characterized by a combination of chalky texture, caused by sphaerocytes in the context, and amyloid spore ornamentation in Melzer's reagent. In some Russulaceae, the context exudes latex when injured (Lebel \& Trappe 2000, Miller et al. 2001, Lebel \& Castellano 2002, Verbeken \& Nuytinck 2013, Verbeken et al. 2014). Russulaceae species form ectomycorrhizal associations with plants (Henkel et al. 2000, Tedersoo \& Nara 2010, Kong et al. 2015, Buyck et al. 2018). This family has often been regarded as a large and complex group that 
includes agaricoid, corticioid, gasteroid, pleurotoid, and secotioid members (Buyck \& Horak 1999, Calonge \& Martín 2000, Larsson \& Larsson 2003, Miller et al. 2006, Larsson 2007). Previous analyses indicated that the transformation from agaricoid ancestors to secotioid and gasteroid species has happened many times in evolutionary history of the Russulaceae (Miller et al. 2001, 2006). Thus, the concept that the secotioid Russulaceae genera are synonyms of the agaricoid ones has been gradually accepted during recent years. Zelleromyces and Arcangeliella were included in Lactarius, while Cystangium, Elasmomyces, Gymnomyces, Macowanites, and Martellia, are secotioid members of Russula (Eberhardt \& Verbeken 2004, Lebel \& Tonkin 2007, Buyck et al. 2010, Verbeken et al. 2014, Sang et al. 2016).

Secotioid Russulaceae species have been thoroughly studied in Australia, New Zealand (Bougher 1997, Bougher \& Lebel 2001, Lebel 2001, 2002, 2003a, b, Lebel \& Castellano 2002), and North America (Zeller \& Dodge 1919, 1936, Singer \& Smith 1960, Smith 1963, Thiers 1984b, Miller \& Lebel 1999, Desjardin 2003, Smith et al. 2006). However, there are fewer records from Southeast Asia (Verbeken et al. 2014) and China (Zeller \& Dodge 1935, Zhang \& Yu 1990, Tao \& Chang 1993, Liu 1998, Li et al. 2012, Sang et al. 2016). In this study, a hypogeous secotioid species Lactarius sulphosmus from the subtropical area of southeast China, was found to be new to science based on morphological characteristics and phylogenetic analysis. Another novel species Russula vinosobrunneola from northeast China is agaricoid in morphology. However, it has a close phylogenetic relationship with the semi-gasteroid species $R$. sichuanensis G.J. Li \& H.A. Wen. These two species are herein described and illustrated in detail. Their morphological and phylogenetic analyses with closely related taxa are also provided.

\section{Materials \& Methods}

\section{Specimens and morphology}

Specimens were photographed and collected in the field and their macroscopic characteristics were recorded at the same time under daylight. Color name and codes followed the standard of Ridgway (1912). Specimens were dehydrated using a Vatti fruit dryer (Zhongshan, China) at $60{ }^{\circ} \mathrm{C}$ overnight. Specimens of the two new species were deposited in the Herbarium Mycologicum, Academiae Sinicae (HMAS). Herbarium name abbreviations followed those of Thiers (2018). Small pieces of dry specimens were rehydrated in $5 \% \mathrm{KOH}$ solution. Rehydrated lamellae and pileus pellis were sliced by hand using a Flying Eagle razor blade (Shanghai, China). Microscopic characters were observed under a Nikon Eclipse 80i optical microscope (Tokyo, Japan). Observations, measurements, and line drawings of basidiospores, basidia, and cystidia elements of the pileipellis and stipitipellis were performed with the help of Congo Red. Melzer's reagent and Sulphovanillin (SV) were employed to test the reactions of basidiospore ornamentations and cystidium contents. The lengths of the apiculus and spore ornamentations were excluded from measurements. At least 50 basidiospores and 20 other elements were measured for each characteristic. Scanning electron microscope (SEM) photos of basidiospores were captured through a Hitachi SU8010 Field Emission SEM (Tokyo, Japan). The abbreviation $[a / b / c]$ represented that $a$ basidiospores were measured from $b$ fruit bodies of $c$ specimens. Basidiospore widths and lengths were displayed as $(w-) x-y(-z)$, where $x-y$ was the range including $95 \%$ or more of the measured values, while $w$ and $z$ were the extremes of all measured values. Q stands for the ratio of basidiospore length/width. A bold $\mathbf{Q}$ stands for the average value of $\mathbf{Q}$ plus or minus the standard deviation. Further details of the microscopic observations in this study are available in Li et al. (2012, 2015), Li (2014).

\section{DNA extraction, polymerase chain reaction (PCR) and sequencing}

Small pieces of dried fruiting bodies were pulverized with a Xinyi 48 tissue grinder (Ningbo, China) by shaking for $80 \mathrm{~s}$ at $30 \mathrm{~Hz} 2$ times in a 2-mL tube with one $3 \mathrm{~mm}$ steel ball. Genomic DNA was extracted using a using a modified magnetic bead method with a GeneOn Plant DNA Extraction Kit (Changchun, China) following the manufacturer's instructions. Gene regions were 
amplified using following pairs of primers: ITS1/ITS4 (White et al. 1990) for the 5.8S and internal transcribed spacers ITS1 and ITS2 regions (ITS), LROR/LR5 (Moncalvo et al. 2000, 2002) for the large subunit of the nuclear ribosomal DNA ( $n L S U$ ), MS1/MS2 (White et al. 1990) for the small subunit of the mitochondrial ribosomal DNA region ( $m t S S U$ ), and EF1-983F/EF1-1567R (Morehouse et al. 2003) for the translation elongation factor alpha (tefl- $\alpha$ ). PCRs were performed in a $50 \mu \mathrm{L}$ reaction mixture that contained the following: $21 \mu \mathrm{L} \mathrm{ddH}_{2} \mathrm{O}, 25 \mu \mathrm{L} 2 \times$ Plus Master Mix (Biomed Biotech Beijing), $1.5 \mu \mathrm{L}$ each primer $(10 \mathrm{mmol} / \mathrm{L})$, and $1 \mu \mathrm{L}$ DNA template, in an ABI Verti 96 Thermal Cycler (Applied Biosystems, Foster City, CA, USA). PCR procedures followed an initial denaturation at $95{ }^{\circ} \mathrm{C}$ for $4 \mathrm{~min}$, followed by 35 cycles of $94{ }^{\circ} \mathrm{C}$ for $50 \mathrm{~s}, 51{ }^{\circ} \mathrm{C}$ for $55 \mathrm{~s}$, $72{ }^{\circ} \mathrm{C}$ for $1 \mathrm{~min}$, and a final extension at $72{ }^{\circ} \mathrm{C}$ for $10 \mathrm{~min}$ for $I T S, n L S U$, and $m t S S U$; an initial denaturation at $95{ }^{\circ} \mathrm{C}$ for $4 \mathrm{~min}$, followed by 15 cycles of $94{ }^{\circ} \mathrm{C}$ for $50 \mathrm{~s}, 65-50{ }^{\circ} \mathrm{C}$ for $55 \mathrm{~s}$ (reduced by $1{ }^{\circ} \mathrm{C}$ per cycle), 20 cycles of $94{ }^{\circ} \mathrm{C}$ for $50 \mathrm{~s}, 50{ }^{\circ} \mathrm{C}$ for $55 \mathrm{~s}, 72{ }^{\circ} \mathrm{C}$ for $1 \mathrm{~min}, 72{ }^{\circ} \mathrm{C}$ for $1 \mathrm{~min}$, and a final extension at $72{ }^{\circ} \mathrm{C}$ for $10 \mathrm{~min}$ for tef- $1 \alpha$. The PCR products were purified and sequenced by Biomed Biotech Company using an ABI 3130 DNA sequencer and the ABI BigDye 3.1 Terminator Cycle Sequencing Kit (Applied Biosystems, Foster City, CA, USA).

\section{Phylogenic analyses}

Raw forward and reverse sequences were assembled using DNAStar v7.1 (Lasergene, WI, USA). GenBank and UNITE accession numbers of ITS sequences are shown in Tables 1, 2. Alignments were performed in Mafft 7.376 (Katoh \& Standley 2013). Necessary edits and adjustments were made using Bioedit (Hall 1999). Ambiguously aligned regions and gaps in matrixes were removed from the analyses with Gblocks 0.91b (Talavera \& Castresana 2007). Sequences for multi-gene analysis were combined in SequenceMatrix 1.8 (Vaidya et al. 2011). Bayesian analysis was conducted in MrBayes 3.2.6 (Ronquist \& Huelsenbeck 2003). The best-fit model of evolution was estimated using MrModelTest 2.3 (Nylander 2004) before analysis. Posterior probabilities (PP) were calculated with the Markov chain Monte Carlo (MCMC) algorithm (Larget \& Simon 1999). Runs of 1,000,000 generations with the tree sampled every 100th generation were initiated for three heated and one cold Markov chains. The analyses were automatically terminated when the average standard deviation of the split frequencies reached a value below 0.01 . The first $25 \%$ of trees were discarded as burn-in. Maximum Likelihood (ML) analysis was performed with RAxMLHPC2 v. 8.2.4 (Stamatakis 2014) as implemented on the CIPRES portal (Miller et al. 2010), using a GTRGAMMA model with 1000 rapid bootstrap (BS) replicates. The alignment matrices for Bayesian and ML analyses were deposited in TreeBASE (ID 22573). The phylogenetic tree was displayed in TreeGraph 2.4.0 (Stöver \& Müller 2010).

Table 1 Specimens and their GenBank/UNITE accession numbers analyzed in ITS phylogenetic analyses. Specimens with newly acquired sequences for this study are shown in bold.

\begin{tabular}{|c|c|c|c|c|}
\hline Taxon & Location & Voucher No. & $\begin{array}{l}\text { Sequence } \\
\text { accession No. }\end{array}$ & Reference \\
\hline Arcangeliella borziana & Switzerland & $\begin{array}{l}\text { WSL (F. Ayer) } \\
\text { Nr. 96-05-3344 }\end{array}$ & AF286204 & $\begin{array}{l}\text { Peter et al. } \\
\text { (2001) }\end{array}$ \\
\hline Arcangeliella borziana & Switzerland & NA & AF373599 & $\begin{array}{l}\text { Peter et al. } \\
(2001)\end{array}$ \\
\hline Arcangeliella camphorata & Japan & OSC 119445 & EU644702 & NA \\
\hline Arcangeliella camphorata & Japan & OSC 74230 & EU644701 & NA \\
\hline Arcangeliella crassa & USA & OSC 41826 & KT968563 & NA \\
\hline Arcangeliella sp. & Thailand & AC7R3 & FJ454900 & Roy et al. (2009) \\
\hline Arcangeliella sp. & Australia: Tasmania & $\mathrm{BH} 2148 \mathrm{~F}$ & JF960610 & Horton (2011) \\
\hline $\begin{array}{l}\text { Gloeocystidiellum } \\
\text { aculeatum }\end{array}$ & China: Taiwan & Wu890714-52 & AF506433 & $\begin{array}{l}\text { Larsson \& Larsson } \\
\text { (2003) }\end{array}$ \\
\hline
\end{tabular}


Table 1 Continued.

\begin{tabular}{|c|c|c|c|c|}
\hline Taxon & Location & Voucher No. & $\begin{array}{l}\text { Sequence } \\
\text { accession } \\
\text { No. }\end{array}$ & Reference \\
\hline $\begin{array}{l}\text { Lactarius aff. } \\
\text { wenquanensis }\end{array}$ & Thailand & GENT LTH143 & EF141537 & Le et al. (2007) \\
\hline Lactarius angiocarpus & Zambia: Copperbelt & $\begin{array}{l}\text { GENT Arora 00- } \\
448\end{array}$ & AY606942 & $\begin{array}{l}\text { Eberhardt \& } \\
\text { Verbeken (2004) }\end{array}$ \\
\hline Lactarius akahatsu & Japan: Tottori & TMI 22601 & EF141556 & Le et al. (2007) \\
\hline $\begin{array}{l}\text { Lactarius } \\
\text { alboscrobiculatus } \\
\text { Lactarius }\end{array}$ & Thailand & GENT LTH175 & EF141538 & Le et al. (2007) \\
\hline $\begin{array}{l}\text { alboscrobiculatus var. } \\
\text { roseopurpureus }\end{array}$ & Thailand & GENT LTH148 & EF141540 & Le et al. (2007) \\
\hline Lactarius alnicola & USA & $\begin{array}{l}\text { MONT EB0064- } \\
14\end{array}$ & KX394276 & $\begin{array}{l}\text { Barge \& Cripps } \\
\text { (2016) }\end{array}$ \\
\hline Lactarius alpinus & Europe & FNL GNP-125 & KX094937 & $\begin{array}{l}\text { Nuytinck \& Voitk } \\
\text { (2016) }\end{array}$ \\
\hline Lactarius austrozonarius & $\begin{array}{l}\text { Thailand: Chiang } \\
\text { Mai }\end{array}$ & GENT FH12-007 & KF432965 & $\begin{array}{l}\text { Wisitrassameewong } \\
\text { et al. (2014) }\end{array}$ \\
\hline $\begin{array}{l}\text { Lactarius } \\
\text { austrotorminosus }\end{array}$ & Thailand & GENT LTH111 & EF141531 & Le et al. (2007) \\
\hline Lactarius camphoratus & Sweden & $\begin{array}{l}\text { UPS } \\
\text { UE04.09.2004-5 }\end{array}$ & DQ422009 & NA \\
\hline Lactarius chichuensis & China: Yunnan & HKAS Wang1236 & KF241541 & $\begin{array}{l}\text { Verbeken et al. } \\
\text { (2014) }\end{array}$ \\
\hline Lactarius chrysorrheus & Italy & $\begin{array}{l}\text { UPS } \\
\text { UE04.10.2002-8 }\end{array}$ & KF133261 & $\begin{array}{l}\text { Verbeken et al. } \\
\text { (2014) }\end{array}$ \\
\hline Lactarius citriolens & Sweden & $\begin{array}{l}\text { UPS } \\
\text { UE20.09.2004-03 }\end{array}$ & DQ422003 & NA \\
\hline Lactarius crassus & USA & Trappe 17996 & KT968581 & NA \\
\hline Lactarius cyathuliformis & Sweden & $\begin{array}{l}\text { UPS } \\
\text { UE04.09.2004-2 }\end{array}$ & KF133266 & $\begin{array}{l}\text { Verbeken et al. } \\
\text { (2014) }\end{array}$ \\
\hline Lactarius deliciosus & Slovakia & $\begin{array}{l}\text { GENT JN2001- } \\
046\end{array}$ & KF133272 & $\begin{array}{l}\text { Verbeken et al. } \\
\text { (2014) }\end{array}$ \\
\hline Lactarius deterrimus & Slovakia & $\begin{array}{l}\text { GENT JN 2001- } \\
053\end{array}$ & DQ922515 & $\begin{array}{l}\text { Nuytinck \& } \\
\text { Verbeken (2007) }\end{array}$ \\
\hline Lactarius echinus & Sri Lanka & AV07-168 & KF133273 & $\begin{array}{l}\text { Verbeken et al. } \\
\text { (2014) }\end{array}$ \\
\hline Lactarius falcatus & Thailand & $\begin{array}{l}\text { GENT KVP08- } \\
038\end{array}$ & KF133274 & $\begin{array}{l}\text { Verbeken et al. } \\
\text { (2014) }\end{array}$ \\
\hline Lactarius formosus & Thailand & GENT LTH382 & EF141549 & Le et al. (2007) \\
\hline Lactarius hatsudake & $\begin{array}{l}\text { Vietnam: Lam } \\
\text { Dong }\end{array}$ & $\begin{array}{l}\text { GENT JN2011- } \\
065\end{array}$ & KF432967 & $\begin{array}{l}\text { Wisitrassameewong } \\
\text { et al. (2014) }\end{array}$ \\
\hline Lactarius helvus & Sweden & $\begin{array}{l}\text { UPS } \\
\text { UE08.09.2004-1 }\end{array}$ & KF133263 & $\begin{array}{l}\text { Verbeken et al. } \\
\text { (2014) }\end{array}$ \\
\hline Lactarius illyricus & Austria & VM 14644 & JF908315 & $\begin{array}{l}\text { Osmundson et al. } \\
\text { (2013) }\end{array}$ \\
\hline Lactarius lignicola & China: Yunnan & $\begin{array}{l}\text { KUN-HKAS } \\
59474\end{array}$ & MF508946 & Wang (2017) \\
\hline $\begin{array}{l}\text { Lactarius pallido- } \\
\text { ochraceus }\end{array}$ & China: Yunnan & $\begin{array}{l}\text { KUN-HKAS } \\
59446\end{array}$ & MF508943 & Wang (2017) \\
\hline Lactarius olympianus & North America & ED 08-018 & KR364089 & $\begin{array}{l}\text { De Crop et al. } \\
(2016)\end{array}$ \\
\hline
\end{tabular}


Table 1 Continued.

\begin{tabular}{|c|c|c|c|c|}
\hline Taxon & Location & Voucher No. & $\begin{array}{l}\text { Sequence } \\
\text { accession } \\
\text { No. }\end{array}$ & Reference \\
\hline Lactarius pallidizonatus & China: Yunnan & $\begin{array}{l}\text { KUN-HKAS } \\
62019\end{array}$ & MF508931 & Wang (2017) \\
\hline Lactarius pomiolens & Sri Lanka & AV07-159 & KF133282 & $\begin{array}{l}\text { Verbeken et al. } \\
\text { (2014) }\end{array}$ \\
\hline Lactarius porninsis & Slovakia & $\begin{array}{l}\text { GENT JN 2001- } \\
082\end{array}$ & DQ922547 & $\begin{array}{l}\text { Nuytinck \& } \\
\text { Verbeken (2007) }\end{array}$ \\
\hline Lactarius psammicola & USA: Michigan & BPL869 & KY848507 & NA \\
\hline Lactarius purpureus & $\begin{array}{l}\text { Thailand: Chiang } \\
\text { Mai }\end{array}$ & GENT FH12-008 & KF432966 & $\begin{array}{l}\text { Wisitrassameewong } \\
\text { et al. (2014) }\end{array}$ \\
\hline Lactarius purpureus & Thailand & GENT LTH120 & EF141542 & Le et al. (2007) \\
\hline Lactarius purpureus & Papua New Guinea & E4552 & EF141543 & Le et al. (2007) \\
\hline Lactarius quietus & Sweden & $\begin{array}{l}\text { UPS } \\
\text { UE16.09.2004 }\end{array}$ & KF133264 & $\begin{array}{l}\text { Verbeken et al. } \\
\text { (2014) }\end{array}$ \\
\hline $\begin{array}{l}\text { Lactarius } \\
\text { repraesentaneus }\end{array}$ & Europe & NA & AY331011 & $\begin{array}{l}\text { Nuytinck et al. } \\
\text { (2003) }\end{array}$ \\
\hline Lactarius rubriviridis & USA & DED7312 & EF685088 & $\begin{array}{l}\text { Nuytinck et al. } \\
\text { (2007) }\end{array}$ \\
\hline Lactarius rufus & Europe & ED2008-06 & KT165278 & $\begin{array}{l}\text { Wisitrassameewong } \\
\text { et al. (2016) }\end{array}$ \\
\hline Lactarius sanguifluus & Belgium & $\begin{array}{l}\text { GENT JN 2000- } \\
008\end{array}$ & AY332547 & $\begin{array}{l}\text { Nuytinck \& } \\
\text { Verbeken (2003) }\end{array}$ \\
\hline Lactarius salmonicolor & Europe & NA & AF140265 & $\begin{array}{l}\text { Eberhardt et al. } \\
(2000)\end{array}$ \\
\hline Lactarius saturnisporus & Sri Lanka & AV07-170 & KF133283 & $\begin{array}{l}\text { Verbeken et al. } \\
\text { (2014) }\end{array}$ \\
\hline Lactarius scrobiculatus & Slovakia & GENT JN01-058 & KF432968 & $\begin{array}{l}\text { Wisitrassameewong } \\
\text { et al. (2014) }\end{array}$ \\
\hline Lactarius speciosus & $\begin{array}{l}\text { USA: North } \\
\text { Carolina }\end{array}$ & AV 04/206 & $\mathrm{EF} 141547$ & Le et al. (2007) \\
\hline Lactarius sphagneti & UK & PL2805 & KF133268 & $\begin{array}{l}\text { Verbeken et al. } \\
\text { (2014) }\end{array}$ \\
\hline Lactarius spinosporus & China: Sichuan & BJTC FAN445 & KY270490 & Sang et al. (2017) \\
\hline Lactarius stephensii & Belgium & RW2930 & AY331012 & $\begin{array}{l}\text { Nuytinck et al. } \\
(2003)\end{array}$ \\
\hline Lactarius subbrevipes & China: Yunnan & $\begin{array}{l}\text { KUN-HKAS } \\
94947\end{array}$ & MF508934 & Wang (2017) \\
\hline Lactarius subdulcis & Belgium & JV2006-024 & KF133279 & $\begin{array}{l}\text { Verbeken et al. } \\
\text { (2014) }\end{array}$ \\
\hline Lactarius subsericatus & Sweden & $\begin{array}{l}\text { UPS } \\
\text { UE11.10.2004-8 }\end{array}$ & DQ422011 & NA \\
\hline Lactarius sulphosmus & China: Fujian & HMAS 279586 & MG819726 & This study \\
\hline Lactarius sulphosmus & China: Fujian & HMAS 276808 & MG719937 & This study \\
\hline Lactarius torminosus & Canada & OUC 97057 & DQ367908 & Durall et al. (2006) \\
\hline Lactarius uvidus & Europe & NA & AY606957 & $\begin{array}{l}\text { Eberhardt \& } \\
\text { Verbeken (2004) }\end{array}$ \\
\hline $\begin{array}{l}\text { Lactarius } \\
\text { vinaceorufescens }\end{array}$ & Canada & $\begin{array}{l}\text { GENT JN2007- } \\
018\end{array}$ & KF241542 & $\begin{array}{l}\text { Verbeken et al. } \\
\text { (2014) }\end{array}$ \\
\hline Lactarius yumthangensis & India & BSHC Das 11-147 & KJ742418 & Das et al. (2015) \\
\hline Russula aurantioflammans & Finland: Enontekiö & TUR A796 & UDB011116 & NA \\
\hline
\end{tabular}


Table 1 Continued.

\begin{tabular}{|c|c|c|c|c|}
\hline Taxon & Location & Voucher No. & $\begin{array}{l}\text { Sequence } \\
\text { accession No. }\end{array}$ & Reference \\
\hline Russula cessans & $\begin{array}{l}\text { Estonia: Hiiu } \\
\text { maakond }\end{array}$ & TU 101805 & UDB015971 & NA \\
\hline Russula cessans & North America & S. Miller 9678 & AY061730 & $\begin{array}{l}\text { Miller \& Buyck } \\
(2002)\end{array}$ \\
\hline $\begin{array}{l}\text { Russula } \mathrm{cf} . \\
\text { aurantioflammans }\end{array}$ & China: Neimenggu & HMAS 252635 & KX441038 & Unpublished \\
\hline Russula cf. fulvograminea & China: Neimenggu & HMAS 267791 & KX441108 & Unpublished \\
\hline Russula cf. graminea & China: Yunnan & HMAS 271166 & KX441221 & Unpublished \\
\hline Russula cf. laricina & China: Liaoning & HMAS 267755 & KX441101 & Unpublished \\
\hline Russula emetica & Germany & TUB lw081 & UDB000300 & NA \\
\hline Russula emetica & United Kingdom & DG44 & UDB001628 & NA \\
\hline Russula font-queri & $\begin{array}{l}\text { Estonia: Valga } \\
\text { maakond }\end{array}$ & TU101814 & UDB015978 & NA \\
\hline Russula font-queri & China: Jilin & HMAS 267744 & KX441096 & Unpublished \\
\hline Russula fulvograminea & Finland: Paltamo & TU 101621 & UDB016259 & NA \\
\hline Russula fulvograminea & $\begin{array}{l}\text { Finland: } \\
\text { Vehmersalmi }\end{array}$ & JR $2810 \mathrm{~F}$ & UDB011112 & NA \\
\hline Russula fulvograminea & China: Jilin & HMAS 252626 & KX441036 & Unpublished \\
\hline Russula laricina & Europe & E Watling 25556 & AY061685 & $\begin{array}{l}\text { Miller \& Buyck } \\
(2002)\end{array}$ \\
\hline Russula laricina & China & HMAS 252564 & KF850405 & Li (2014) \\
\hline Russula laricina & Europe & $575 / 08.681$ & JN944008 & $\begin{array}{l}\text { Schoch et al. } \\
\text { (2012) }\end{array}$ \\
\hline Russula laricina & China: Sichuan & HMAS 269630 & KX441153 & Unpublished \\
\hline Russula nauseosa & $\begin{array}{l}\text { Estonia: Saare } \\
\text { maakond }\end{array}$ & TU 118126 & UDB011239 & NA \\
\hline Russula nauseosa & $\begin{array}{l}\text { Estonia: Saare } \\
\text { maakond }\end{array}$ & TU 106370 & UDB011164 & NA \\
\hline Russula nauseosa & $\begin{array}{l}\text { Estonia: Tartu } \\
\text { maakond }\end{array}$ & TU 101692 & UDB011360 & NA \\
\hline Russula nauseosa & United Kingdom & DG 05-47 & UDB001716 & NA \\
\hline Russula nauseosa & $\begin{array}{l}\text { Estonia: Pärnu } \\
\text { maakond }\end{array}$ & TU 101789 & UDB011333 & NA \\
\hline Russula nauseosa & Europe & FH 12173 & KT933985 & $\begin{array}{l}\text { Looney et al. } \\
\text { (2016) }\end{array}$ \\
\hline Russula nauseosa & Chian: Guizhou & MYH 2013100345 & KX655853 & $\mathrm{NA}$ \\
\hline Russula sichuanensis & China: Sichuan & HMAS 268888 & KX441125 & Unpublished \\
\hline Russula sichuanensis & China: Sichuan & HKAS 53885 & JX391968 & Li et al. (2013) \\
\hline Russula sichuanensis & China: Sichuan & HKAS 53792 & JX391969 & Li et al. (2013) \\
\hline Russula sichuanensis & China: Gansu & HMAS 255316 & MG786566 & This study \\
\hline Russula sp. & China: Heilongjiang & HMJAU 32220 & KX095013 & NA \\
\hline Russula sp. & China: Heilongjiang & HMJAU 32153 & KX095024 & NA \\
\hline Russula sp. & China: Heilongjiang & HMJAU 32222 & KX095022 & NA \\
\hline Russula sp. & China: Yunnan & HKAS 78378 & KF002768 & Guo et al. (2014) \\
\hline Russula sp. & China: Heilongjiang & HMJAU 32217 & KX094991 & NA \\
\hline Russula sp. & China: Heilongjiang & HMJAU 32216 & KX094990 & NA \\
\hline Russula sp. & China: Yunnan & HKAS 78371 & KF002761 & Guo et al. (2014) \\
\hline Russula sp. & China: Yunnan & HKAS 78377 & KF002767 & Guo et al. (2014) \\
\hline Russula sp. & China: Yunnan & HKAS 78370 & KF002760 & Guo et al. (2014) \\
\hline
\end{tabular}


Table 1 Continued.

\begin{tabular}{|c|c|c|c|c|}
\hline Taxon & Location & Voucher No. & $\begin{array}{l}\text { Sequence } \\
\text { accession } \\
\text { No. }\end{array}$ & Reference \\
\hline Russula vinosobrunneola & $\begin{array}{l}\text { China: } \\
\text { Heilongjiang }\end{array}$ & HMAS 278896 & MG719923 & This study \\
\hline Russula vinosobrunneola & $\begin{array}{l}\text { China: } \\
\text { Heilongjiang }\end{array}$ & HMAS 281131 & MG719924 & This study \\
\hline Russula vinosobrunneola & $\begin{array}{l}\text { China: } \\
\text { Heilongjiang }\end{array}$ & HMAS 278885 & MG719925 & This study \\
\hline Russula vinosobrunneola & $\begin{array}{l}\text { China: } \\
\text { Heilongjiang }\end{array}$ & HMAS 278960 & MG719926 & This study \\
\hline Russula vinosobrunneola & $\begin{array}{l}\text { China: } \\
\text { Heilongjiang }\end{array}$ & $\begin{array}{l}\text { HMAS } 281138 \\
\text { (Type) }\end{array}$ & MG719927 & This study \\
\hline Russula violaceoincarnata & Finland & UPS2005.09.06-11 & UDB002549 & NA \\
\hline Zelleromyces cinnabarinus & USA & FLAS-F-60604 & MH016796 & NA \\
\hline Zelleromyces gardneri & USA & NA & DQ453696 & $\begin{array}{l}\text { Frank et al. } \\
(2006)\end{array}$ \\
\hline Zelleromyces gardneri & USA & NA & JN022500 & NA \\
\hline Zelleromyces giennensis & Spain & NA & AF230900 & $\begin{array}{l}\text { Calonge \& Martín } \\
(2000)\end{array}$ \\
\hline Zelleromyces hispanicus & Spain & MA-Fungi 37497 & AF231911 & $\begin{array}{l}\text { Calonge \& Martín } \\
(2000)\end{array}$ \\
\hline Zelleromyces hispanicus & Spain & MA-Fungi 37498 & AF231912 & $\begin{array}{l}\text { Calonge \& Martín } \\
(2000)\end{array}$ \\
\hline Zelleromyces sp. & $\begin{array}{l}\text { Australia: } \\
\text { Tasmania }\end{array}$ & BH3528R & JF960852 & Horton (2011) \\
\hline
\end{tabular}

Table 2 Specimens and their GenBank accession numbers analyzed in multi-gene phylogenetic analyses. Specimens with newly acquired sequences for this study are shown in bold.

\begin{tabular}{llllll}
\hline Taxon & Voucher No. & LSU & ITS & tef-1a & mtSSU \\
& & & & & \\
\hline Russula cf. aurantioflammans & HMAS 252635 & KX441285 & KX441038 & MF893370 & KX441532 \\
Russula cf. fulvograminea & HMAS 267791 & KX441355 & KX441108 & MF893440 & KX441602 \\
Russula cf. graminea & HMAS 271166 & KX441468 & KX441221 & MF893553 & KX441715 \\
Russula cf. laricina & HMAS 267755 & KX441348 & KX441101 & MF893433 & KX441595 \\
Russula cf. melliolens sp.1 & HMAS 269070 & KX441378 & KX441131 & MF893463 & KX441625 \\
Russula cf. melliolens sp.2 & HMAS 276813 & LT602938 & LT602961 & KX442503 & LT602984 \\
$\begin{array}{l}\text { Russula font-queri } \\
\text { Russula fulvograminea }\end{array}$ & HMAS 267744 & KX441343 & KX441096 & MF893428 & KX441590 \\
$\begin{array}{l}\text { Russula laricina } \\
\text { Russula sichuanensis }\end{array}$ & HMAS 269630 & KX441400 & KX441153 & MF893485 & KX441647 \\
Russula sichuanensis & HMAS 268888 & KX441372 & KX441125 & MF893457 & KX441619 \\
Russula vinosobrunneola & HMAS 278896 & MG786567 & MG719923 & MG812155 & MG792318 \\
Russula vinosobrunneola & HMAS 278960 & MG786568 & MG719926 & MG812156 & MG792319 \\
Russula vinosobrunneola & HMAS 281138 & MG786569 & MG719927 & MG812157 & MG792320 \\
Russula vinosobrunneola & HMAS 278885 & MG786570 & MG719925 & MG812158 & MG792321 \\
Russula vinosobrunneola & HMAS 281131 & MG786571 & MG719924 & MG812159 & MG792322 \\
\hline
\end{tabular}




\section{Results}

\section{Phylogenetic analyses}

For Lactarius: The best-fit model of Bayesian analysis was GTR $+\mathrm{I}+\mathrm{G}$. The Maximum Likelihood and Bayesian trees had almost identical topology except for several minor terminal rearrangements, which were not statistically supported. The new species $L$. sulphosmus clustered with L. illyricus Piltaver in section Zonarii (Quél.) Bon in Lactarius subgenus Piperites (Fr. ex J. Kickx fil.) Kauffman (BS 76\%, PP 0.98, Fig. 1). A blast against the GenBank database showed that the ITS sequence of the new species has $95 \%$ identity with those of L. illyricus, L. purpureus R. Heim, and L. psammicola A.H. Sm. The topologies generally agree with those described by Le et al. (2007) and Verbeken et al. (2014). The monophyly of both subgenus Piperites, sections Colorati (Bataille) Hesler \& A.H. Sm., Uvidi (Konrad) Bon, Piperites (Fr. ex J. Kickx fil.) Burl., and Zonarii was not supported.

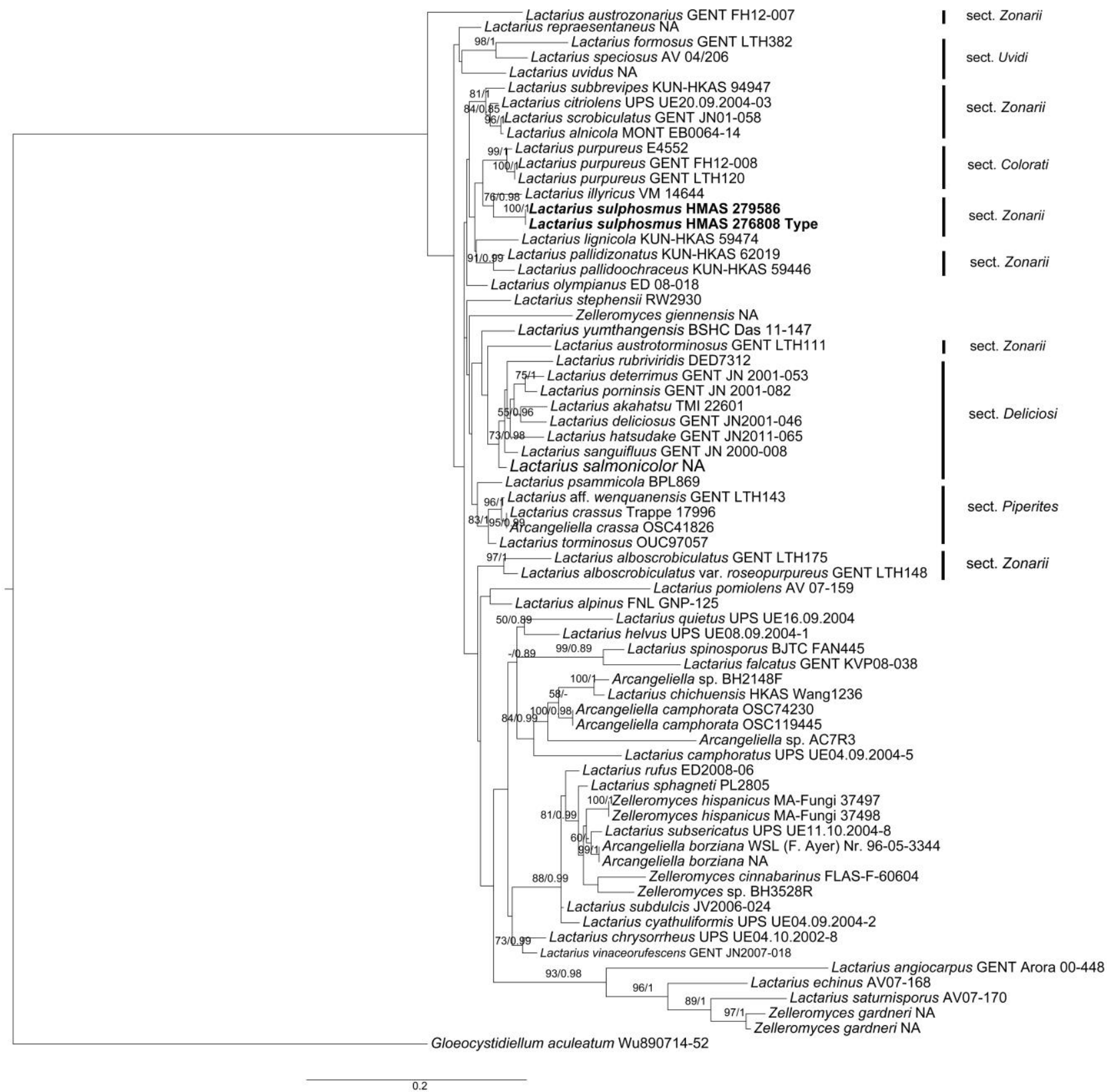

Figure 1 - Phylogeny of Lactarius subgenus Piperites and other closely related species generated from the Maximum Likelihood analysis of the ITS sequences. Bootstrap support (BS) $>50 \%$ and Bayesian posterior probability (PP) values $>0.8$ are given at the internodes (BS / PP). 
For Russula: The best-fit models of Bayesian analyses were HKY (ITS1), HKY+G (ITS2 and ITS1-5.8S-ITS2), GTR+I+G ( $n L S U$ and 5.8S), F81+G (mtSSU), and SYM+G (tef-1 $\alpha)$. The ITSbased phylogeny of Russula section Tenellae (Quél.) Sarnari subsection Laricinae (Romagn.) Bon was unable to distinguish three common species $R$. nauseosa, $R$. laricina, and $R$. cessans in Clade A (Fig. 2), which had poor statistical support (BS 52\%, PP-). Clade B was highly supported by 96\% BS and 1 PP values (Fig. 2). It included the agaricoid species $R$. vinosobrunneola, the semigasteroid species $R$. sichuanensis G.J. Li \& H.A. Wen, some unrecognized Russula taxa from China, and $R$. nauseosa (TU 118126 from Estonia). Although the morphological differences of $R$. sichuanensis and $R$. vinosobrunneola were remarkable, the ITS-based phylogeny could not separate them. Russula font-queri Singer, $R$. aurantioflammans Ruots., Sarnari \& Vauras, $R$. fulvograminea Ruots., Sarnari \& Vauras, $R$. violaceoincarnata Knudsen \& T. Borgen, and three more taxa $(R$. cf. aurantioflammans, $R$. cf. fulvograminea, and $R$. cf. graminea) formed a clade that was supported by a PP value of 0.77 (Clade C, Fig. 2).

Therefore, a multi-gene phylogenetic analysis of $n L S U, I T S, m t S S U$, and tef- $1 \alpha$ was carried out for a better specific level resolution. The multi-gene phylogenetic topology (Fig. 3) showed that $R$. vinosobrunneola and $R$. sichuanensis were separated into two clades with strong support ( $R$. vinosobrunneola: BS 98\%, PP 0.99, R. sichuanensis: BS 100\%, PP 1.00).

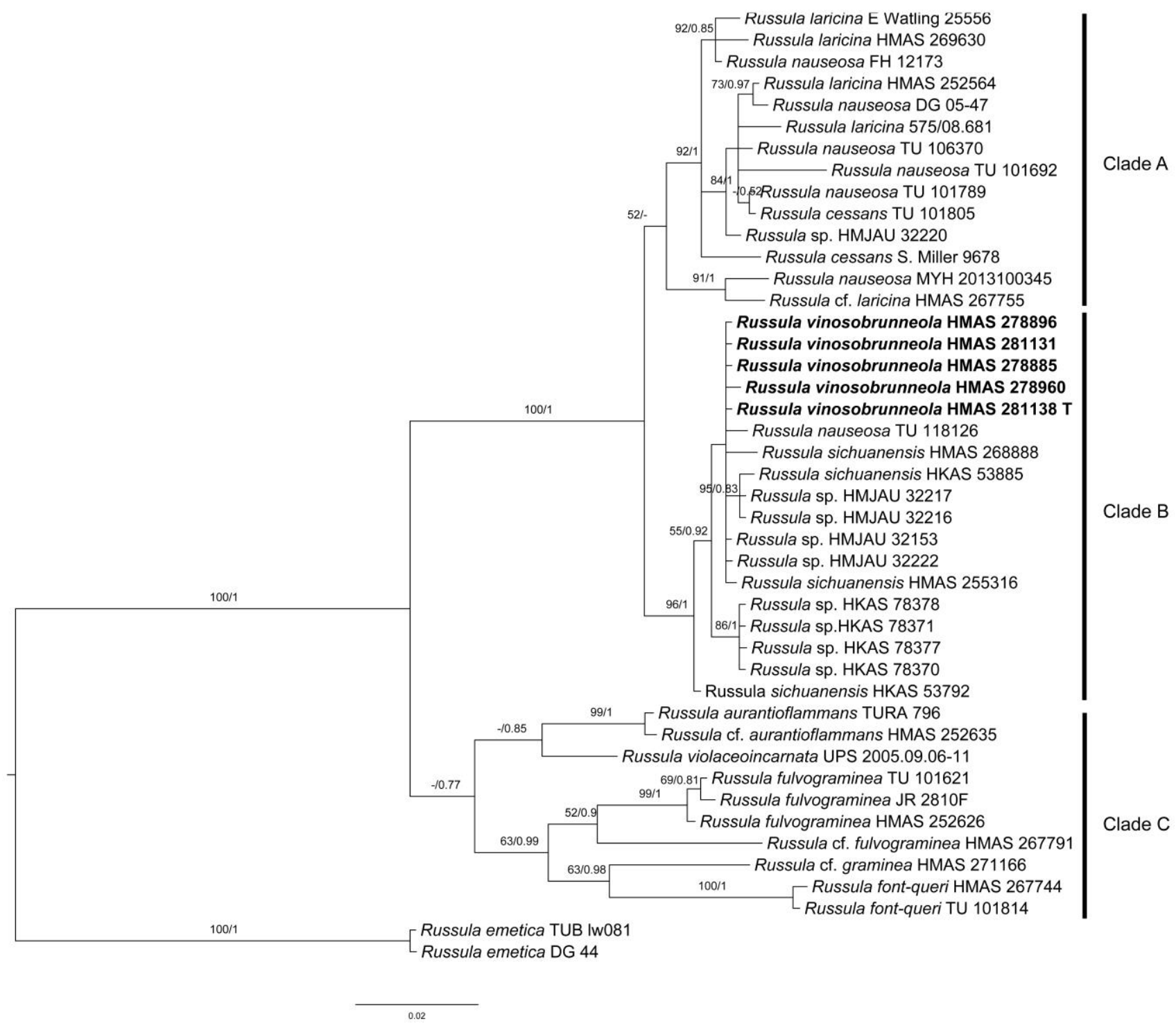

Figure 2 - Phylogeny of Russula section Tenellae subsection Laricinae generated from the Bayesian analysis of the ITS sequences. Bootstrap support (BS) values > $50 \%$ and Bayesian posterior probability $(\mathrm{PP})$ values $>0.8$ are given at the internodes (BS / PP). 


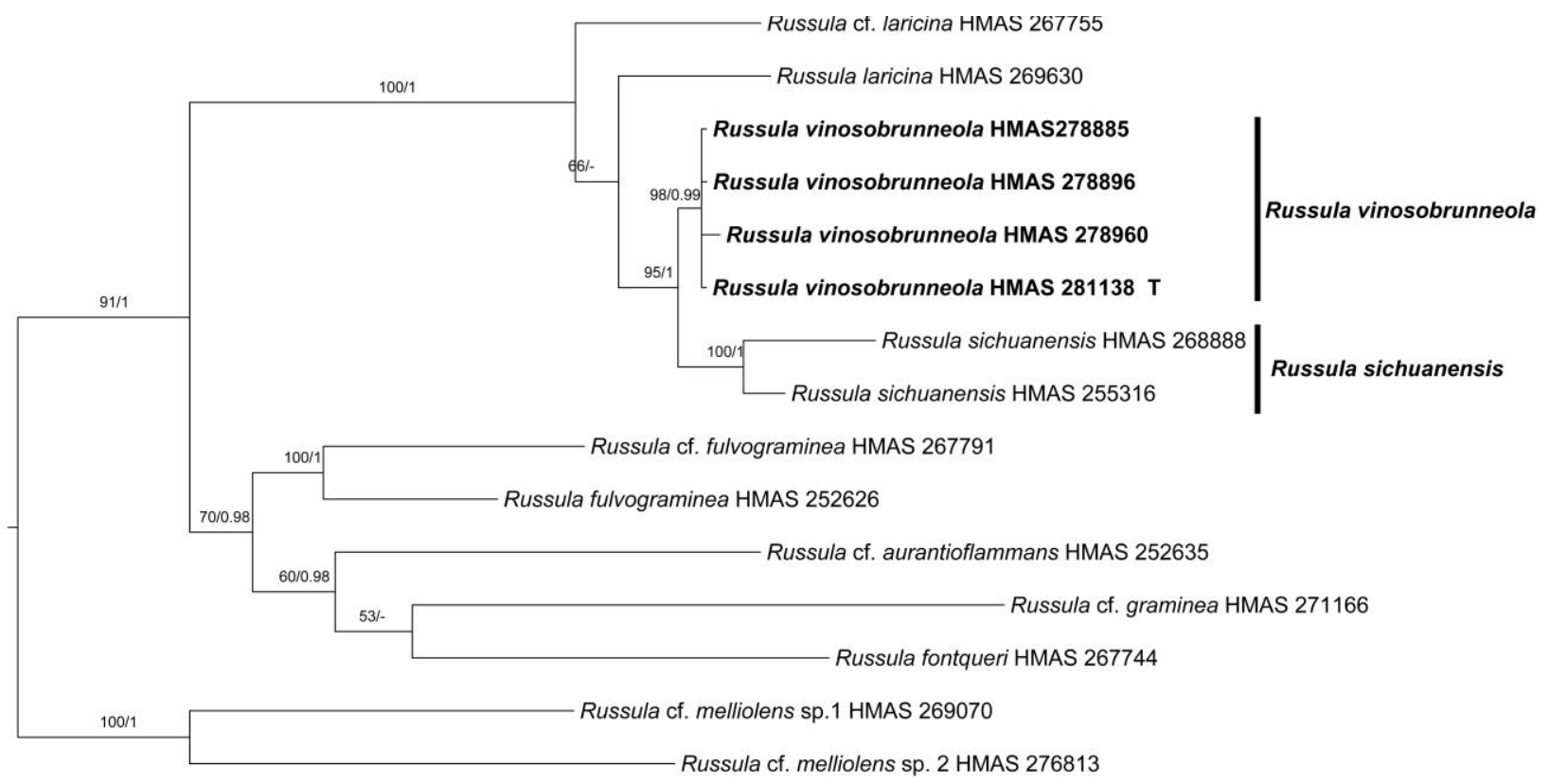

Figure 3 - Phylogeny of $R$. vinosobrunneola and $R$. sichuanensis and allied species generated from the Bayesian analysis of a combination of ITS, $n L S U, m t S S U$ and tef- $1 \alpha$ gene sequences. Bootstrap support (BS) values $>50 \%$ and Bayesian posterior probability $(\mathrm{PP})$ values $>0.8$ are given at the internodes (BS / PP).

\section{Taxonomy}

Lactarius sulphosmus G.J. Li \& R.L. Zhao, sp. nov.

MycoBank number: MB824979; Facesoffungi number: FoF03950

Figs $4 \mathrm{a}-\mathrm{b}, 5$

Etymology - refers to the strongly sulphide-like smell of basidiomata.

Typus - China, Fujian Province, Wuyishan City, Wuyishan National Nature Reserve, Huangxizhou, N27 $43^{\prime}$ E117²6', elevation 823 m, 6 June 2015, collector Rui Lin Zhao, Guo Jie Li, Mao Qiang He, and Sheng Yu Su, ZRL20150189. (HMAS 276808).

Diagnosis - Basidiomata $35-46 \times 25-31 \mathrm{~mm}$, irregularly subglobose. Peridial surface pale yellow, dull. Gleba cream, loculate. Columella absent. Latex abundant. Smell strongly like sulphide, reminiscent of hair burning. Basidiospores globose to subglobose, with isolated and curved spines branched at apex. Basidia four-spored. Pseudocystidia rare. True cystidia rare.

Description - Basidiomata small to medium-sized, 35-46 $\times 25-31 \mathrm{~mm}$, irregularly globose to subglobose, planoconcave at base. Peridium irregularly shaped, often with furrows up to $5 \mathrm{~mm}$ in depth, smooth, dull, not viscid when wet, pale yellowish tinged, light buff (XV17') to pale ochraceous-buff (XV15'f), often intermixed with a darker ocher tinge of chamois (XXX19'b) to honey yellow (XXX19"). Stipe and columella absent. Gleba first compact, crowdedly loculate when mature, rarely labyrinthoid (2-3 per mm), cream colored, cartridge buff (XXX19"f) to ivory yellow (XXX21"f), partly turning ochraceous tawny (XV15'i) to honey yellow (XXX19"). Latex white, rather abundant, thick and sticky, mild tasting, forming a thin and white layer on gleba when dry. Smell strongly like sulphide, reminiscent of hair burning. Taste mild.

Basidiospores [500/2/2] (11.3-) 12.3-15.4 (-15.9) × (10.5-) 11.2-13.3 (-14) $\mu \mathrm{m}, \mathrm{Q}=1.00$ 1.25 (-1.28) $(\mathrm{Q}=1.14 \pm 0.07)$, globose to subglobose, rarely broadly ellipsoid, echinulate; ornamentation composed of amyloid warts that are isolated, curved, rounded, or shortly double or triple forked at the apex, 1.8-2.2 $\mu \mathrm{m}$ in height; suprahilar area amyloid but indistinct. Basidia 25$29 \times 6-8 \mu \mathrm{m}$, four-spored, rarely two-spored, sterigmata 1-2 $\mu \mathrm{m}$ long, subcylindrical to cylindrical, rarely subclavate, hyaline, unchanging in $\mathrm{KOH}$. True cystidia rare, 32-43 $\times 9-11 \mu \mathrm{m}$, clavate to subclavate, sometimes fusoid, subfusoid to subcylindrical, apex rounded to obtuse, thin-walled, 
hollow, unchanging in SV. Pseudocystidia rare, cylindrical, 4-6 $\mu \mathrm{m}$ in diameter (diam.) Peridiopellis a trichoderm, composed of thin-walled, mostly ascending to suberect, unbranched, cylindrical hyaline hyphae 3-6 $\mu \mathrm{m}$ wide; pileocystidia absent; terminal cells cylindrical, apex round to obtuse, rarely tapered. Clamp connections absent in all tissues.

Habit and habitat - Hypogeous, single in broad-leaved forest (dominated by e.g., Castanopsis eyrie, C. faberi, C. fargesii, Eurya japonica, Lithocarpus hancei, Machilus thunbergii, Manglietia fordiana, and Schima superba) at 800-900 m altitude.

Other specimens examined - China, Fujian Province, Wuyishan City, Wuyishan National

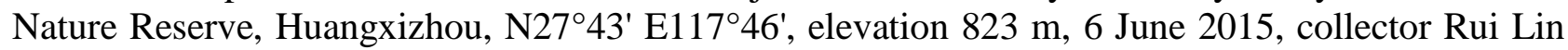
Zhao, Guo Jie Li, Mao Qiang He and Sheng Yu Su, ZRL20154537. (HMAS 279586).

Distribution - China (Fujian Province). Season. June.

Notes - The combination of abundant white latex, strong sulphidic, burning hair smell, spore ornamentation composed of high spine with double or triple forked tips, is distinct for the new species L. sulphosmus. The ITS-based phylogenetic analysis (Fig. 1) showed that L. sulphosmus and L. illyricus from Slovenia form a clade (BS 76\%, PP 0.98), which indicates that they are closely related in phylogeny. However, they are obviously different species in morphology, since $L$. illyricus is an agaricoid species (Le et al. 2007, Piltaver 1992). This new species has pale yellow basidiomata, not hairy pileus and white, unchanging latex, which are characters of section Zonarii (Bon 1980).

In morphology L. sulphosmus is most similar to the Chinese species Zelleromyces ramispinus (B.C. Zhang \& Y.N. Yu) Trappe, T. Lebel \& Castellano (basionym: Martellia ramispina B.C. Zhang \& Y.N. Yu) because both have gasteroid basidiomata, spore surface with spines over $2 \mu \mathrm{m}$ high and double or triple forked tips, and a habitat of subtropical forest. However, Z. ramispinus has white peridium surface, and larger basidiospores (15-20 $\mu \mathrm{m}$ in diam, Zhang \& Yu 1990).

Russula vinosobrunneola G.J. Li \& R.L. Zhao, sp. nov.

Figs $4 \mathrm{c}-\mathrm{d}, 6$

MycoBank number: MB824980; Facesoffungi number: FoF04080

Etymology - refers to the vinaceous brown tinged, small to medium-sized pileus.

Typus - China, Heilongjiang Province, Qitaihe City, Boli County, Xidaquan National Forest Park, N 454' E 130³4', elv. 753 m., 19 July 2016, Ming-Zhe Zhang, Xu-Ming Bai, Rong-Chun Dai, Guo-Jie Li, ZRL20160434. (HMAS 281138).

Diagnosis - the morphological characteristics of $R$. vinosobrunneola are similar to those of $R$. laricina, but $R$. vinosobrunneola has amyloid ornamentation, composed of warts mostly linked by fine lines as ridges, reticulate, rarely isolated, forming an incomplete to complete reticulum.

Description - Basidiomata small to medium-sized. Pileus 11-54 $\mathrm{mm}$ in diam., first subglobose to hemispheric, plano-convex when mature, sometimes depressed at center, slightly striate about 3-5 mm from the edge inwards when old, rarely cracked, slightly viscid when wet, sometimes glabrous, peeling 1/5-1/3 from the edge, often vinaceous brown tinged, hazel (XIV11'k), liver brown (XIV7'm) to vinaceous russet (XXVIII7") at the edge, a paler tinge of avellaneous (XL17"'b), fawn color (XL13"') to olive buff (XL21'"'d) towards the center. Lamellae adnate, 2-4 $\mathrm{mm}$ in height, 11-16 per $\mathrm{cm}$ at the edge, not forked, often interveined, first pale yellowish tinges of martius yellow (III23f), turning Apricot Yellow (XL21"'d) to Light Cadmium (IV19) when mature, Orange-Citrine (IV19k) to Medal Bronze (III19m) when old and dry, lamellulae absent. Stipes central to subcentral, $4.2-6.5 \times 0.9-1.6 \mathrm{~cm}$, subcylindrical to cylindrical, surface dry, rugulose longitudinally, dull, without annulus, slightly attenuate upwards, White (LIII), a brown tinge of Tawny (XV13'i) when bruised and dry, stuffed at first, becoming hollow when old. Context up to $1-3 \mathrm{~mm}$ at the center of the pileus, White (LIII), unchanging, slowly turning greyish brown, tinges of Sudan Brown (III15k), Ochraceous-Tawny (XV15'i) to Buckthorn Brown (XV17'i) when bruised, fragile; odor not distinct; taste mild. Spore print Yellow (Romagnesi IV bc).

Basidiospores [500/2/2] 7.7-9.6 (-10.1) × 6.4-8 (-8.6) $\mu \mathrm{m}, \mathrm{Q}=1.06-1.33(-1.38)(\mathrm{Q}=1.19$ \pm 0.07 ), subglobose to broadly ellipsoid, rarely globose to ellipsoid, verrucose; ornamentation 
amyloid, composed of verrucose to conical warts $0.7-1 \mu \mathrm{m}$ in height, mostly linked by fine lines as ridges, reticulate, rarely isolated, forming an incomplete to complete reticulum; suprahilar area amyloid and distinct. Basidia 34-42 × 8-11 $\mu \mathrm{m}$, clavate, ventricose towards upper half, fourspored, projecting 15-25 $\mu \mathrm{m}$ beyond hymenium, hyaline in $\mathrm{KOH}$; sterigmata 4-6 $\mu \mathrm{m}$, pointed, straight to slightly tortuous. Pleurocystidia scattered, 47-72 $\times 6-7 \mu \mathrm{m}$, originating from subhymenium, projecting 20-35 $\mu \mathrm{m}$ beyond hymenium, thin-walled, subfusiform to subclavate, sometimes clavate or fusiform, apex obtuse to round, at times subacute, contents crystalline, blackish grey in SV. Cheilocystidia not observed; lamellar edge sterile. Subhymenium 20-50 $\mu \mathrm{m}$ thick, composed of slender cylindrical cells 4-10 $\mu \mathrm{m}$ wide under basidia and inflated elliptical to globose cells $15-25 \mu \mathrm{m}$ in diam. next to trama. Pileipellis 150-270 $\mu \mathrm{m}$ thick, composed of an epipellis (60-90 $\mu \mathrm{m}$ thick) and a subpellis (90-230 $\mu \mathrm{m}$ thick); epipellis a cutis or trichoderm, composed of mostly ascending to repent hyphae, intermixed with a few suberect elements, $2-4 \mu \mathrm{m}$ in diam.; pileocystidia abundant, 37-55 $\times 3-5 \mu \mathrm{m}$, cylindrical to subcylindrical, with round to obtuse tips, multi-septate, contents dense, black in SV; subpellis composed of long, rarely septate, equally wide hyphae 2-4 $\mu \mathrm{m}$ in diam. interweaved with sphaerocytes $15-30 \mu \mathrm{m}$ in diam. Stipitipellis a cutis composed of thin-walled, septate, cylindrical, hyaline to slightly yellowish hyphae 3-5 $\mu \mathrm{m}$ wide; terminal cells of stipitipellis hyphae with obtuse apex; caulocystidia not observed. Trama composed of sphaerocystes $15-60 \mu \mathrm{m}$ in diam. and septate filamentous hyphae. Clamp connections and lacticiferous hyphae absent in all tissues.
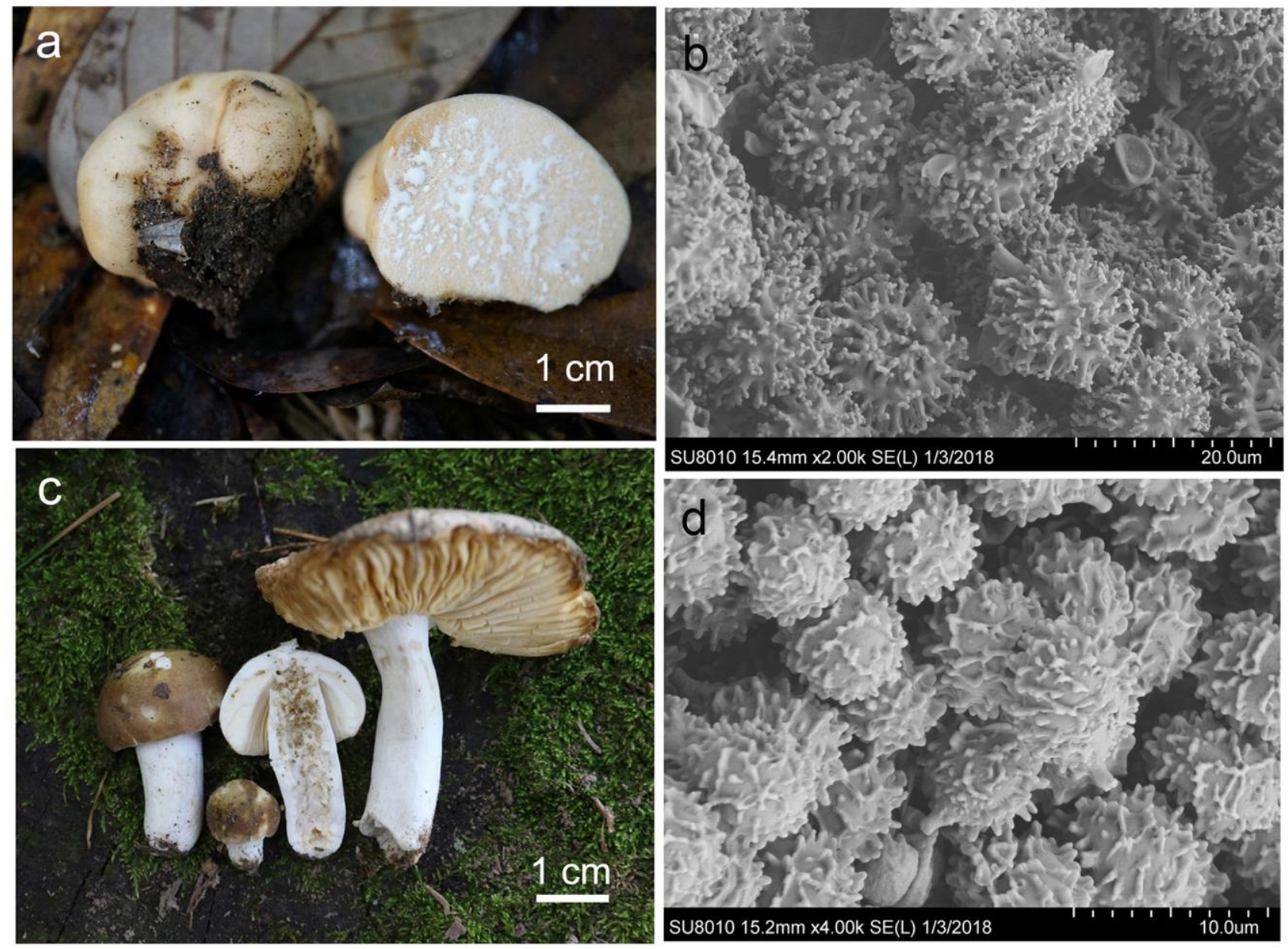

Figure 4 - Basidiomata and scanning electron micrograph (SEM) of basidiospores. a-b Lactarius sulphosmus: ZRL20150189 (HMAS 276808 holotype). c-d Russula vinosobrunneola: ZRL20160434 (HMAS 281138 holotype). 

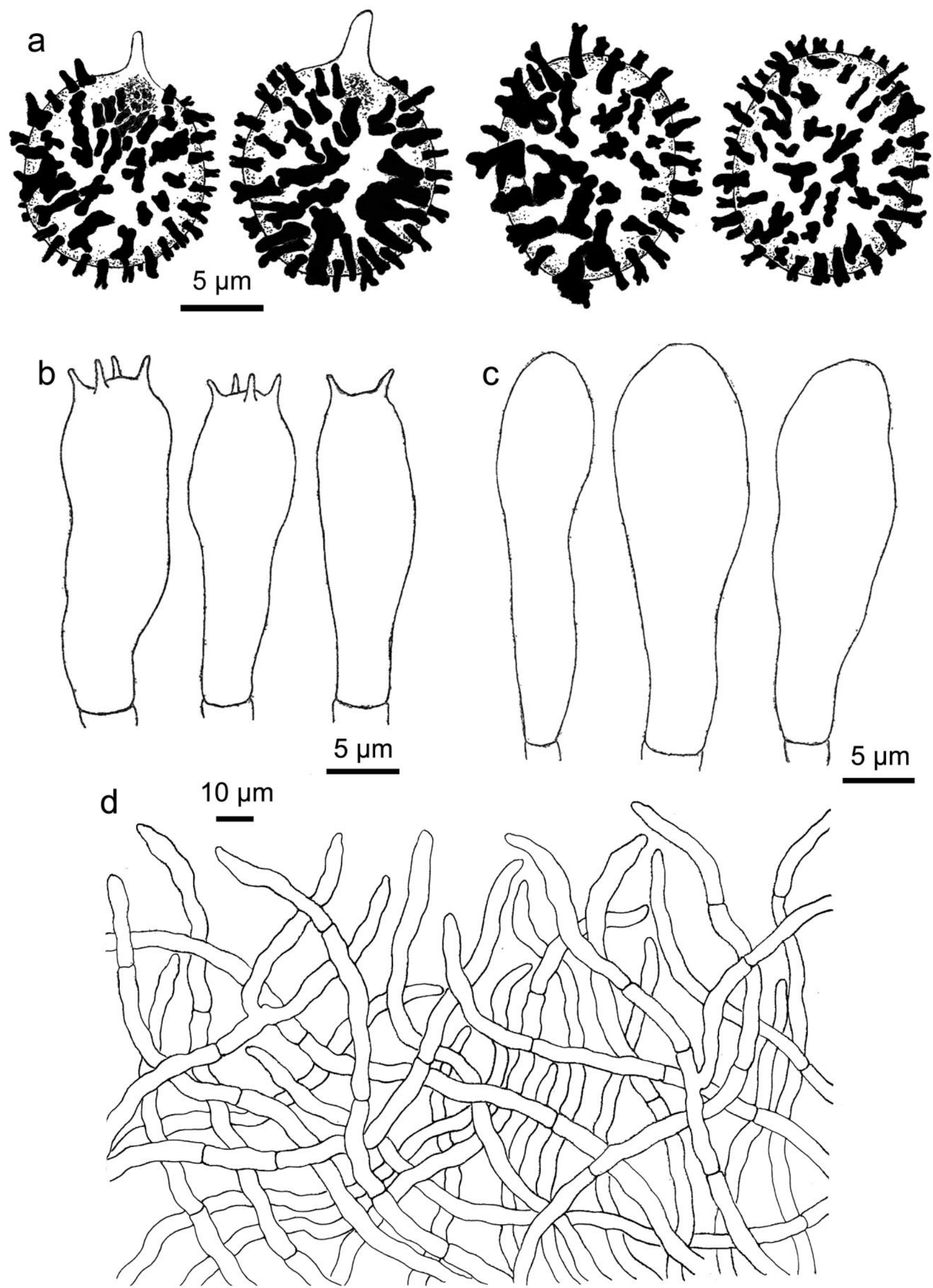

Figure 5 - Lactarius sulphosmus: ZRL20150189 (HMAS 276808 holotype) a Basidiospores. b Basidia. c True cystidia. d Pileipellis (epipellis). 
Habit and habitat - Scattered in coniferous and broad-leaved intermixed forests (dominated by e.g., Abies nephrolepis, Pinus bungeana, P. koraiensis, P. sylvestris var. mongolica, Tilia tuan, and Xylosma racemosum) at 600-800 m altitude.

Other specimens examined - China, Heilongjiang Province, Kiamusze City, Tangyuan

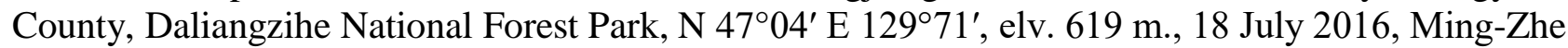
Zhang, Xu-Ming Bai, Rong-Chun Dai, Guo-Jie Li, ZRL20160383. (HMAS 281131); Qitaihe City, Boli County, Xidaquan National Forest Park, N 4545' E 130³4', elv. 827 m., 19 July 2016, MingZhe Zhang, Xu-Ming Bai, Rong-Chun Dai, Guo-Jie Li, ZRL20160428. (HMAS 278960); elv. 721 m., ZRL20160394. (HMAS 278885); Yichun City, Dailing District, Liangshui National Nature Reserve, N 47 $18^{\prime}$ E $128^{\circ} 87^{\prime}$, elv. 657 m., 17 July 2016, Rui-Lin Zhao, Ming-Zhe Zhang, Xu-Ming Bai, Rong-Chun Dai, Guo-Jie Li, ZRL20160273. (HMAS 278896).

Distribution - China (Heilongjiang Province). Season. July.

Notes - A combination of yellow spore print, greyish brown turning context, and coniferous habitat indicates that $R$. vinosobrunneola is a member of Russula section Tenellae subsection Laricinae (Sarnari 2005). The semi-gasteroid species $R$. sichuanensis is the closest species to $R$. vinosobrunneola phylogenetically, but they can be readily distinguished because $R$. sichuanensis has secotioid basidiomata, dirty-white to pale-pinkish pileus, globose to subglobose, larger basidiospores $8.8-15.5 \times 7.6-13.1 \mu \mathrm{m}$ with warts often higher than $1 \mu \mathrm{m}$, shorter and wider basidia 24-35 × 11-15 $\mu \mathrm{m}$, and an ixotrichoderm pileipellis ( $\mathrm{Li}$ et al. 2013). Compared with other members of subsect. Laricinae, $R$. vinosobrunneola most resembles $R$. laricina Velen., due to the similar pileus colors and dimensions of basidiospores, basidia, and pleurocystidia. The only difference is that the basidiospore ornamentation of $R$. laricina is composed of mostly isolated warts (Sarnari 2005), while those of $R$. vinosobrunneola is mostly reticulum. The basidiospore ornamentation reticulum from this new species is also helpful to separate it from $R$. nauseosa (Pers.) Fr. (Sarnari 2005). Although $R$. cessans A. Pearson has similar basidiospore ornamentations to those of $R$. vinosobrunneola, it can be distinguished for having pink to vinaceous pileus, subacrid and unchanging context, and wider pleurocystidia up to $12 \mu \mathrm{m}$. The other uncommon species of $R$. subsect. Laricinae, such as $R$. olivina Ruots. \& Vauras, differ from $R$. vinosobrunneola in their two-spored basidia and larger basidiospores of 10-14 $\times 8-11.5 \mu \mathrm{m}$ and $R$. sapinea Sarnari differs from $R$. vinosobrunneola by its strongly yellowish context and ocher spore print (Sarnari 2005). Russula vinosobrunnea (Bres.) Romagn., a species of $R$. section Polychromidia Romagn., subsection Olivaceinae Singer, is taken for $R$. vinosobrunneola because they both have vinaceous brown pileus, yellow spore prints, and basidiospores $7-10 \times 6-8 \mu \mathrm{m}$. The similarity of specific epithets can also lead to the confusion of these two species. However, $R$. vinosobrunnea can be distinguished by its large stout basidiomata with pileus up to $120 \mathrm{~mm}$ in diam., long pleurocystidia up to $130 \mu \mathrm{m}$ in length, pileipellis with primodial hyphae without incrustation, and a habitat of Fagus forest (Sarnari 2005).

\section{Discussion}

The coexistence of agaricoid, secotioid, and gasteroid lineages within one group has been reported many times in Agricomycetes (Wilson et al. 2011). The phylogenetic relationships between the agaricoid and gasteroid lineages have been analyzed in many studies (Hopple \& Vilgalys 1994, Mueller \& Pine 1994, Kretzer \& Bruns 1997, Miller et al. 2001, Wilson et al. 2011) and agaricoid basidiomata has been shown to be the plesiomorphy. The agaricoid new species of this study $R$. vinosobrunneola has a close phylogenetic relationship with secotioid $R$. sichuanensis (Fig. 2). Russula sichuanensis is distributed in coniferous forests of the Qinghai-Tibet Plateau (Li et al. 2013, Saba \& Khalid 2015). The differences between the habitats of these two species corroborate the viewpoint that the stage of gasteromycetization is a coping mechanism for the hostile environment (Hibbett et al. 1994, Buyck 1995, Trappe \& Claridge 2005, Smith et al. 2006, Verbeken et al. 2014). The results of phylogenetic analyses also showed that these two species could not be distinguished by ITS phylogeny, indicating the speciation may have occurred recently in their evolutionary history. 

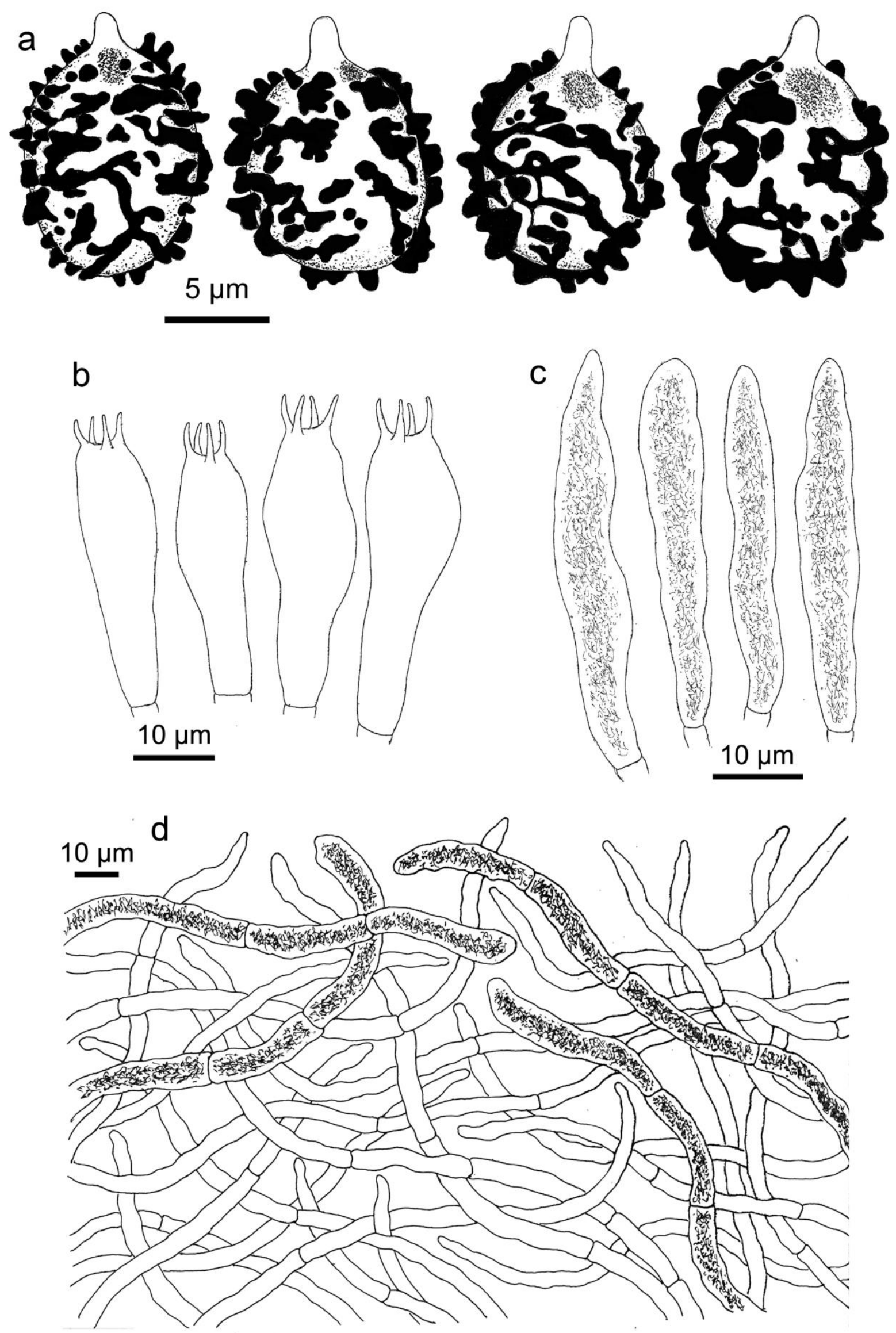

Figure 6 - Russula vinosobrunneola: ZRL20160434 (HMAS 281138 holotype) a Basidiospores. b Basidia. c True cystidia. d Pileipellis (epipellis). 
Verbeken et al. (2014) revealed a striking diversity of sequestrate Russulales in Southeast Asia. A total of ten species have been recognized in this area. In China, most species of sequestrate Russulales, including L. sulphosmus of this study, are described from subtropical regions. Only Zelleromyces sinensis B. Liu, K. Tao \& Ming C. Chang is from a temperate region in China (Tao \& Chang 1993). Compared with the recent morphological descriptions of the agaricoid species in detail (Buyck \& Adamčík 2013, Buyck et al. 2013, 2015, Adamčík et al. 2015a, 2018), those of above angiocarpous taxa are often brief. Considering that angiocarpous mushrooms are often overlooked (Verbeken et al. 2014), a majority of recent new Russula species are agaricoid, whatever their collecting localities are in Africa (Sanon et al. 2014), Asia (Das et al. 2013, 2017a, 2017b, 2018, Ariyawansa et al. 2015, Hyde et al. 2016, Li et al. 2016, 2018b, Jiang et al. 2017, Song et al. 2018), America (Adamčík et al. 2015b, Hyde et al. 2017), and Europe (Trendel et al. 2017). Thus further investigations of gasteroid species are more needed than agaricoid ones. As divergence time can be used as a universal criterion for taxa ranking (Zhao et al. 2016, 2017). We suggest that the divergence times of the agaricoid, secotioid, and gasteroid Russulaceae lineages can be estimated in future evolution analyses. Because it is not only helpful in evolutionary relationship clarification these lineages, but also useful in the systematic analyses of corresponding species.

\section{Acknowledgements}

This work was financed by the National Natural Science Foundation of China (Project IDs GJL: 31500013, RLZ: 31470152 and 31360014) and the National Project on Scientific Ground work for Basic Science of the Ministry of Science and Technology (No. 2014FY210400). Yan-Lei Ding (Inner Mongolia University of Science \& Technology, Baotou Normal College), Zi-Kun Xu (The management Bureau of Wuyishan National Natural Reserve of Fujian), Mao-Qiang He, Sheng-Yu Su (Southwest Forestry University), An-Qi Liu, Zhi-Lin Ling, Xu-Ming Bai, Ming-Zhe Zhang, and Rong-Chun Dai are thanked for their helps in specimen collection. The authors are grateful to Liu Yang for loans of herbarium specimens; Chun-Li Li, and Xiao-Lan Zhang (Institute of Microbiology, Chinese Academy of Sciences) for providing helps with SEM photography; XinYu Zhu and Bing-Jing Di (Inner Mongolia University of Science \& Technology, Baotou Normal College) for helps in DNA extraction and sequencing.

\section{References}

Adamčík S, Jančovičová S, Buyck B. 2015a - Type-studies in American Russula subsection Decolorantes (Russulales, Basidiomycota), part II. Phytotaxa 321(3), 245-259.

Adamčík S, Cai L, Chakraborty D, Chen XH, et al. 2015b - Fungal biodiversity profiles 1-10. Cryptogamie Mycologie 36(2), 121-166.

Adamčík S, Jančovičová S, Buyck B. 2018 - The Russulas described by Charles Horton Peck. Cryptogamie Mycologie 39(1), 3-108.

Ariyawansa HA, Hyde KD, Jayasiri SC, Buyck B et al. 2015 - Fungal diversity notes 111-252 taxonomic and phylogenetic contributions to fungal taxa. Fungal Diversity 75(1), 27-274.

Barge EG, Cripps CL. 2016 - New reports, phylogenetic analysis, and a key to Lactarius Pers. in the Greater Yellowstone Ecosystem informed by molecular data. MycoKeys 15, 1.

Binder M, Bresinsky A. 2002 - Derivation of a polymorphic lineage of Gasteromycetes from boletoid ancestors. Mycologia 94(1), 85-98.

Bon M. 1980 - Clé monographique du genre Lactarius (Pers. ex Fr.) S.F. Gray. Documents Mycologiques 10(40), 1-85.

Bougher N. 1997 - Three new sequestrate Basidiomycetes from Western Australia. Mycotaxon 63, $37-48$.

Bougher NL, Lebel T. 2001 - Sequestrate (truffle-like) fungi of Australia and New Zealand. Australian Systematic Botany 14, 439-484.

Bruns TD, Fogel R, White TJ, Palmer JD. 1989 - Accelerated evolution of a false-truffle from a mushroom ancestor. Nature 339, 140-142. 
Buyck B. 1995. - A global integrated approach on the taxonomy of Russulales. Russulales News 3, $2-17$.

Buyck B, Horak E. 1999 - New taxa of pleurotoid Russulaceae. Mycologia 91(3), 532-537.

Buyck B, Hofstetter V, Verbeken A, Walleyn R. 2010 - Proposal to conserve Lactarius nom. cons. (Basidiomycota) with conserved type. Taxon 59, 447-453.

Buyck B, Adamčík S. 2013 - Type studies in Russula subsection Lactarioideae from North America and a tentative key to North American species. Cryptogamie Mycologie 34(4), 367391.

Buyck B, Carteret X, Buyck B. 2013 - Type studies on some Russula species described by C.H. Peck. Cryptogamie Mycologie 34(4), 367-391.

Buyck B, Jančovičová S, Adamčík S. 2015 - The study of Russula in the western United States. Cryptogamie Mycologie 36(2), 193-211.

Buyck B, Zoller S, Hofstetter V. 2018 - Walking the thin line... ten years later: the dilemma of above- versus below-ground features to support phylogenies in the Russulaceae (Basidiomycota). Fungal Diversity 89, 267-292.

Calonge FD, Martín MP. 2000 - Morphological and molecular data on the taxonomy of Gymnomyces, Martellia and Zelleromyces (Elasmomycetaceae, Russulales). Mycotaxon 76, 9-15.

Danks M, Lebel T, Vernes K. 2010 - 'Cort short on a mountaintop' - Eight new species of sequestrate Cortinarius from sub-alpine Australia and affinities to sections within the genus. Persoonia 24(1), 106-126.

Das K, Atri NS, Buyck B. 2013 - Three new species of Russula (Russulales) from India. Mycosphere 4(4), 722-732.

Das K, Ghosh A, Baghela A, Avchar R et al. 2018 - Morphological and phylogenetic evidence for Russula butyroindica sp. nov. (Russulaceae) from the Indian Himalaya. Nova Hedwigia 106(3-4), 295-303.

Das K, Ghosh A, Bhatt RP, Chakraborty D et al. 2017a - Fungal biodiversity profiles 31-40. Cryptogamie Mycologie 38(3), 353-406.

Das K, Ghosh A, Bhatt RP, Chakraborty D et al. 2017b - Fungal biodiversity profiles 41-50. Cryptogamie Mycologie 38(4), 527-547.

Das K, Verbeken A, Nuytinck J. 2015 - Morphological description and phylogenetic placement of four new species of Lactarius from Himalayan India. Mycotaxon 130(1), 105-130.

De Crop E, Nuytinck J, Van de Putte K, Wissitrassameewong K et al. 2016 - A multi-gene phylogeny of Lactifluus (Basidiomycota, Russulales) translated into a new infrageneric classification of the genus. Persoonia 38, 58-80.

Desjardin D. 2003 - A unique ballistosporic hypogeous sequestrate Lactarius from California. Mycologia 95, 1: 148-155.

Eberhardt U, Oberwinkler F, Verbeken A, Rinaldi A et al. 2000 - Lactarius ectomycorrhizae on silver fir (Abies alba): morphological description, molecular characterization, and taxonomic remarks. Mycologia 92, 860-873.

Eberhardt U, Verbeken A. 2004 - Sequestrate Lactarius species from tropical Africa: $L$. angiocarpus sp. nov. and L. dolichocaulis comb. nov. Mycological Research 108(9), 10421052.

Frank JL, Barry S, Southworth D. 2006 - Mammal mycophagy and dispersal of mycorrhizal inoculum in Oregon white oak woodlands. Northwest Science 80, 264-273.

Guo JY, Karunarathna SC, Mortimer PE, Xu JC et al. 2014 - Phylogenetic diversity of Russula from Xiao-Zhongdian, Yunnan, China, inferred from internal transcribed spacer sequence data. Chiang Mai Journal of Science 41(4), 811-821.

Hall TA. 1999 - BioEdit: a user-friendly biological sequence alignment editor and analysis program for Windows 95/98/NT. Nucleic Acids Symposium Series 41, 95-98.

Henkel T, Aime MC, Miller SL. 2000 - Systematics of pleurotoid Russulaceae from Guyana and Japan, with notes on their ectomycorrhizal status. Mycologia 92 (6), 1119-1132. 
Henkel TW, Smith ME, Aime MC. 2010 - Guyanagaster, a new wood-decaying sequestrate fungal genus related to Armillaria (Physalacriaceae, Agaricales, Basidiomycota). American Journal of Botany 97(9), 1474.

Hibbett DS, Tsuneda A, Murakami S. 1994 - The secotioid form of Lentinus tigrinus; genetics and development of a fungal morphological innovation. American Journal of Botany 81, 466487.

Hopple JS Jr, Vilgalys R. 1994 - Phylogenetic relationships among coprinoid taxa and allies based on data from restriction site mapping of nuclear rDNA. Mycologia 86, 96-107.

Horton B. 2011 - Eucalypt decline and ectomycorrhizal fungal community ecology of Eucalyptus delegatensis forest, Tasmania, Australia. Phd Dissertation, University of Tasmania, Hobart.

Hongsanan S, Hyde KD, Bahkali AH, Camporesi E et al. 2015 - Fungal biodiversity profiles 11-20. Cryptogamie Mycologie 36(3), 355-380.

Hyde KD, Chada N, Abreu VP, Bazzicalupo A et al. 2017 - Fungal diversity notes 603-708: taxonomic and phylogenetic notes on genera and species. Fungal Diversity 87(1), 1-235.

Hyde KD, Hongsanan S, Jeewon R, Bhat DJ et al. 2016 - Fungal Diversity notes 367-490: taxonomic and phylogenetic contributions to fungal taxa. Fungal Diversity 80(1), 1-270.

Jiang XM, Li YK, Liang JF, Wu JR. 2017 - Russula brunneovinacea sp. nov., from northeastern China. Mycotaxon 132, 789-797.

Katoh K, Standley DM. 2013 - MAFFT multiple sequence alignment software version 7: improvements in performance and usability. Molecular Biology and Evolution 30(4), 772780.

Kong A, Cifuentes J, Estrada-Torres A, Guzmán-Dávalos L et al. 2015 - Russulaceae associated with mycoheterotroph Monotropa uniflora (Ericaceae) in Tlaxcala, Mexico: a phylogenetic approach. Cryptogamie Mycologie 36(4), 479-512.

Kretzer A, Bruns TD. 1997 - Molecular revisitation of the genus Gastrosuillus. Mycologia 89, 586-589.

Larget B, Simon DL. 1999 - Markov chain Monte Carlo algorithms for the Bayesian analysis of phylogenetic trees. Molecular Biology and Evolution 16, 750-759.

Larsson E, Larsson KH. 2003 - Phylogenetic relationships of russuloid basidiomycetes with emphasis on aphyllophoralean taxa. Mycologia 95(6), 1037-1065.

Larsson KH. 2007 - Re-thinking the classification of corticioid fungi. Mycological Research 111(9), 1040-1063.

Le HT, Nuytinck J, Verbeken A, Lumyong S et al. 2007 - Lactarius in Northern Thailand: 1. Lactarius subgenus Piperites. Fungal Diversity 24, 173-224.

Lebel T. 2001 - A new species of Zelleromyces (Russulales) from Australia. Australasian Mycologist 20(2), 4-8.

Lebel T. 2002 - Sequestrate Russulales of New Zealand: Gymnomyces and Macowanites. New Zealand Journal of Botany 40, 489-509.

Lebel T. 2003a - Australian sequestrate (truffle-like) fungi. XIII. Cystangium (Russulales, Basidiomycota). Australian Systematic Botany 16, 371-400.

Lebel T. 2003b - Australian sequestrate (truffle-like) fungi. XIV. Gymnomyces (Russulales, Basidiomycota). Australian Systematic Botany 16, 401-426.

Lebel T, Castellano MA. 2002 - Type studies of sequestrate Russulales II. Australian and New Zealand species related to Russula. Mycologia 94, 327-354.

Lebel T, Tonkin JE. 2007 - Australasian species of Macowanites are sequestrate species of Russula (Russulaceae, Basidiomycota). Australian Systematic Botany 20, 355-381.

Lebel T, Trappe JM. 2000 - Taxonomic studies in the sequestrate Russulales part I. generic type studies. Mycologia 92, 1188-1205.

Lebel T, Vellinga EC. 2013 - Description and affinities of a sequestrate Lepiota (Agaricaceae) from Australia. Mycological Progress 12(3), 525-532.

Li GJ. 2014 - Taxonomy of Russula from China. Ph.D. dissertation. Institute of Microbiology, Chinese Academy of Sciences \& University of Chinese Academy of Sciences, Beijing. 
Li GJ, Deng DJ, Wei JK, Zhang CL et al. 2018a - Protubera beijingensis sp. nov. (Protophallaceae, Phallales) from China. Phytotaxa 348(2), 133-140.

Li GJ, Hyde KD, Zhao RN, Hongsanan S et al. 2016 - Fungal diversity notes 253-366: taxonomic and phylogenetic contributions to fungal taxa. Fungal Diversity 78(1), 1-237.

Li GJ, Li SF, Liu XZ, Wen HA. 2012 - Russula jilinensis sp. nov. (Russulaceae) from northeast China. Mycotaxon 120, 49-58.

Li GJ, Zhang CL, Zhao RL, Lin FC. 2018b - Two new species of Russula from Northeast China. Mycosphere 9(3), 431-443.

Li GJ, Zhao D, Li SF, Wen HA. 2015 - Russula chiui and R. pseudopectinatoides, two new species from southwestern China supported by morphological and molecular evidence. Mycological Progress $14,33$.

Li GJ, Zhao Q, Zhao D, Li SF et al. 2013 - Russula atroaeruginea and R. sichuanensis spp. nov. from southwest China. Mycotaxon 124, 173-188.

Liu B. 1998 - Flora fungorum Sinicorum. Vol. 7: Hymenogasterales, Melanogasterales and Gautieriales. Science Press, Beijing.

Looney BP, Ryberg M, Hampe F, Sánchez-García M et al. 2016 - Into and out of the tropics: global diversification patterns in a hyperdiverse clade of ectomycorrhizal fungi. Molecular Ecology 25(2), 630-647.

Miller MA, Pfeiffer W, Schwartz T. 2010 - Creating the CIPRES science gateway for inference of large phylogenetic trees in Proceedings of the Gateway Computing Environments Workshop (GCE), 14 Nov. 2010, New Orleans, LA, 1-8.

Miller SL, Buyck B. 2002 - Molecular phylogeny of the genus Russula in Europe with a comparison of modern infrageneric classifications. Mycological Research 106(3), 259-276.

Miller SL, Larsson E, Larsson KH, Verbeken A et al. 2006 - Perspectives in the new Russulales. Mycologia 98(6), 960-970.

Miller SL, Lebel T. 1999 - Hypogeous fungi from the Southeastern United States. II. The genus Zelleromyces. Mycotaxon 72, 15-25.

Miller SL, McClean TM, Walker JF, Buyck B. 2001 - A molecular phylogeny of the Russulales including agaricoid, gasteroid and pleurotoid taxa. Mycologia 93(2), 344-354.

Moncalvo JM, Lutzoni FM, Rehner SA, Johnson J et al. 2000 - Phyligenetic relationships of Agaric fungi based on nuclear large subunit ribosomal DNA sequences. Systematic Biology 49, 278-305.

Moncalvo JM, Vilgalys R, Redhead SA, Johnson JE et al. 2002 - One hundred and seventeen clades of euagarics. Molecular Phylogenetic and Evolution 23, 357-400.

Morehouse EA, James TY, Ganley ARD, Vilgalys R et al. 2003 - Multilocus sequence typing suggests the chytrid pathogen of amphibians is a recently emerged clone. Molecular Ecology $12,395-403$

Mueller GM, Pine EM. 1994 - Evidence for monophyly of Hydnangium, Laccaria, and Podohydnangium based on rDNA sequence data. McIlvainea 11, 61-74.

Nuytinck J, Verbeken A. 2003 - Lactarius sanguifluus versus Lactarius vinosus — molecular and morphological analyses. Mycological Progress 2(3), 227-234.

Nuytinck J, Verbeken A. 2007 - Species delimitation and phylogenetic relationships in Lactarius section Deliciosi in Europe. Mycological Research 111(11), 1285-1297.

Nuytinck J, Verbeken A, Delarue S, Walleyn R. 2003 - Systematics of European sequestrate lactarioid Russulaceae with spiny spore ornamentation. Belgium Journal of Botany 136(2), $145-153$.

Nuytinck J, Verbeken A, Miller SL. 2007 - Worldwide phylogeny of Lactarius section Deliciosi inferred from ITS and glyceraldehyde-3-phosphate dehydrogenase gene sequences. Mycologia 99(6), 820-832.

Nuytinck J, Voitk A. 2016 - Lactarius alpinus. Omphalina 7(3), 12-14.

Nylander JAA. 2004 - MrModelTest v2. Program distributed by the author. Evolutionary Biology Centre, Uppsala University, Uppsala. 
Orihara T, Smith ME, Ge ZW, Maekawa N. 2012 - Rossbeevera yunnanensis (Boletaceae, Boletales), a new sequestrate species from southern China. Mycotaxon 120(1), 139-147.

Peter M, Buchler U, Ayer F, Egli S. 2001 - Ectomycorrhizas and molecular phylogeny of the hypogeous russuloid fungus Arcangeliella borziana. Mycological Research 105, 1231-1238.

Piltaver A. 1992 - Lactarius illyricus sp. nov. from Slovenia. Mycological Research 96(8), 694696.

Ridgway R. 1912 - Color standards and color nomenclature. Robert Ridgway, Washington.

Ronquist F, Huelsenbeck JP. 2003 - MRBAYES 3: Bayesian phylogenetic inference under mixed models. Bioinformatics 19, 1572-1574.

Roy M, Watthana S, Stier A, Richard F et al. 2009 - Two mycoheterotrophic orchids from Thailand tropical dipterocarpacean forests associate with a broad diversity of ectomycorrhizal fungi. BMC Biology 7, 51.

Saba M, Khalid AN. 2015 - Russula sichuanensis and its ectomycorrhizae from Himalayan moist temperate forests of Pakistan. Mycotaxon 130, 629-639.

Sang XY, Li XD, Wang YW, Fan L. 2016 - Four new sequestrate species of Russulaceae found in China. Phytotaxa 289(1), 101-117.

Sanon E, Guissou KML, Yorou NS, Buyck B. 2014 - Le genre Russula au Burkina Faso (Afrique de l'Ouest): Quelques Espèces Nouvelles de Couleur Brunâtre. Cryptogamie Mycologie 35(4), 377-397.

Sarnari M. 2005 - Monografia illustrate de genere Russula in Europa. Tomo Secondo. AMB, Centro Studi Micologici, Trento.

Schoch CL, Seifert KA, Huhndorf S, Robert V et al. 2012 - Nuclear ribosomal internal transcribed spacer (ITS) region as a universal DNA barcode marker for fungi. Proceedings of the National Academy of Sciences of the United States of America 109, 6241-6246.

Singer R, Smith AH. 1960 - Studies on secotiaceous fungi - IX. The astrogastraceous series. Memoirs of the Torrey Botanical Club 21, 1-112.

Smith AH. 1963 - New astrogastraceous fungi from the Pacific Northwest. Mycologia 55, 421441.

Smith ME, Trappe JM, Rizzo DM, Miller S. 2006 - Gymnomyces xerophilus sp. nov. (sequestrate Russulaceae), an ectomycorrhizal associate of Quercus in California. Mycological Research $110,575-582$.

Song Y, Li JW, Buyck B, Zheng JF et al. 2018 - Russula verrucospora sp. nov. and $R$. xanthovirens sp. nov., two novel species of Russula (Russulaceae) from southern China. Cryptogamie Mycologie 39(1), 129-142.

Stamatakis A. 2014 - Raxml version phylogenetic analysis post-analysis large phylogenies. Bioinformatics 30(9), 1312-1313.

Stöver BC, Müller KF. 2010 - TreeGraph 2: Combining and visualizing evidence from different phylogenetic analyses. BMC Bioinformatics 11, 7.

Talavera G, Castresana J. 2007 - Improvement of phylogenies after removing divergent and ambiguously aligned blocks from protein sequence alignments. Systematic Biology 56, 564577.

Tao K, Chang MC. 1993 - New species and new record of hypogeous fungi from China. IV. Acta Mycologia Sinica 12, 103-106.

Tedersoo L, Nara K. 2010 - General latitudinal gradient of biodiversity is reversed in ectomycorrhizal fungi. New Phytologist 185(2), 351-354.

Trappe JM, Claridge AW. 2005 - Hypogeous fungi: evolution of reproductive and dispersal strategies through interactions with animals and mycorrhizal plants. In: Dighton J, Oudemans P, White J (eds), The Fungal Community: 599-623. CRC Press, Boca Raton, Florida.

Trendel JM, Hampe F, Verbeken A. 2017 - Russula vinosofavescens sp. nov., from deciduous forests of Northern Alsace, France. Mycotaxon 132, 707-721. 
Thiers B. 2018 - (continuously updated) Index Herbariorum: A global directory of public herbaria and associated staff. New York Botanical Garden's Virtual Herbarium. New York Botanical Garden, New York. http://sweetgum.nybg.org/ih/ (Accessed 31 January 2018)

Thiers HD. 1984a - The secotioid syndrome. Mycologia 76, 1-8.

Thiers HD. 1984b - The genus Arcangeliella Cav. in the western United States. Sydowia 37, 296308.

Trierveiler-Pereira L, Meijer AAR, Hosaka K, Silveira RMB. 2014 - Updates on Protubera (Protophallaceae, Phallales) and additional notes on P. maracuja. Mycoscience 55, 35-42.

Vaidya G, Lohman DJ, Meier R. 2011 - SequenceMatrix: concatenation software for the fast assembly of multi-gene datasets with character set and codon information. Cladistics 27(2), 171-180.

Verbeken A, Nuytinck J. 2013 - Not every milkcap is a Lactarius. Scripta Botanica Belgica 51, $162-168$.

Verbeken A, Stubbe D, Putte KVD, Eberhardt U et al. 2014 - Tales of the unexpected: angiocarpous representatives of the Russulaceae in tropical South East Asia. Persoonia 32, $13-24$.

Wang XH. 2017 - Seven new species of Lactarius subg. Lactarius (Russulaceae) from southwestern China. Mycosystema 36(11), 1463-1482.

Watling R, Martín MP. 2003 - A sequestrate Psilocybe from Scotland. Botanical Journal of Scotland 55(2), 245-257.

Wilson AW, Binder M, Hibbett D. 2011 - Effects of gasteroid fruiting body morphology on diversification rates in three different clades of fungi estimated using binary state speciation and extinction analysis. Evolution 65(5), 1305-1322.

Wisitrassameewong K, Looney BP, Le HT, De Crop E et al. 2016 - Lactarius subgenus Russularia (Basidiomycota, Russulales): novel Asian species, worldwide phylogeny and evolutionary relationships. Fungal Biology 120 (12), 1554-1581.

Wisitrassameewong K, Nuytinck J, Hyde KD, Verbeken A. 2014 - Lactarius subgenus Russularia (Russulaceae) in Southeast Asia: 1. Species with very distant gills. Phytotaxa 158(1), 23-42.

White TJ, Bruns T, Lee S, Taylor J. 1990 - Amplification and direct sequencing of fungal ribosomal RNA genes for phylogenies. In: Innis MA, Gelfand DH, Sninsky JJ, White TJ (eds) PCR protocols, a guide to methods and applications. Academic, San Diego.

Zeller SM, Dodge CW. 1919 - Arcangeliella, Gymnomyces, and Macowanites in North America. Annals of the Missouri Botanical Garden 6, 49-59.

Zeller SM, Dodge CW. 1935 - New species of Hydnangiaceae. Annals of the Missouri Botanical Garden 22, 365-373.

Zeller SM, Dodge CW. 1936 - Arcangeliella, Gymnomyces, and Macowanites. Annals of the Missouri Botanical Garden 23, 500-638.

Zhang BC, Yu YN. 1990 - Two new species of gasteroid Russulales from China, with notes on taxonomy of Gymnomyces, Martellia and Zelleromyces. Mycological Research 94(4), 457462.

Zhao RL, Li GJ, Sánchez-Ramírez S, Stata M et al. 2017 - A six-gene phylogenetic overview of Basidiomycota and allied phyla with estimated divergence times of higher taxa and a phyloproteomics perspective. Fungal Diversity 84, 43-74.

Zhao RL, Zhou JL, Chen J, Margaritescu S et al. 2016 - Towards standardizing taxonomic ranks using divergence times - a case study for reconstruction of the Agaricus taxonomic system. Fungal Diversity 78, 239-292. 